\title{
Optimization Image Quality of Knee MRI Sagital Plane T2 Weighted TSE Sequences with Variations of Echo Train Length (ETL) on Cartesian and Blade Technique
}

\author{
Rini Indrati $^{1^{*}}$, Dwi Rochmayanti ${ }^{1}$, Heny Rubiyanti ${ }^{2}$, Donny Susilawardhono ${ }^{2}$, Siti Daryati ${ }^{1}$, \\ Sri Mulyati ${ }^{1}$ \\ ${ }^{1}$ Radiodiagnostic and Radiotheraphy Department, Poltekkes Kemenkes Semarang, \\ Indonesia, \\ ${ }^{2}$ Radiology Depertment, Dr. Ramelan Navy Hospital, Surabaya, Indosesia
}

\begin{abstract}
The use of Cartesian and Blade techniques also affects image quality. The Cartesian technique is more commonly used in Knee MRI examinations. Cartesian technique is vulnerable to movement, giving rise to motion artifacts. This motion artifact is often caused by too long scanning time, so ETL settings are important. An alternative to reducing this risk is to use the Blade technique and by setting the appropriate ETL. The research objective was to determine the differences image quality and anatomical information and to determine the technique that produced quality images and MRI anatomical information on the sagittal knee section between the Cartesian ETL and Blade T2 Weighted TSE combination. This is experiment research. Samples of 10 volunteers, carried out scanning with Cartesian and Blade techniques with variations ETL 14, 16, 18. Focus of assessment on ACL, PCL, meniscus, fluid, and fat. Image quality includes SNR and CNR. The assessment of the anatomical information by a radiologist. Data analyzed with Anova, Friedman, and Wilcoxon test. The results study showed that overall, there are significant differences in image quality between Cartesian and Blade techniques with p-value 0.005. There are differences in anatomical information with $\mathrm{p}$-value $<0.001$. The best technique from image quality (SNR and CNR) is Cartesian-ETL 14 and based on anatomical information, the best technique is using Blade-ETL 14. Image quality in the two techniques with ETL 14 there is no significant difference with $\mathrm{p}$-value of 0.414 , so that Cartesian and Blade-ETL 14 settings produce the same image quality.
\end{abstract}

Keywords. Knee MRI, T2 Turbo Spin Echo (TSE), Cartesian, Blade, Echo Train Lenght (ETL).

\footnotetext{
* Corresponding author: riniindrati@poltekkes-smg.ac.id
} 


\section{Introduction}

Knee has a significant contribution to the activities of everyone in daily life. Trauma to the knee joint can cause distal fractures, tibia plateau, ligament rupture and tears in the meniscus $[1,2]$. Many occur in sports, especially football and basketball players [2]. Conventional radiography is the initial examination if there is interference with the knee but can only show joint space and bone [3].

Along with the development of the technological age also influenced the development of medical imaging, which until now has become important to support the diagnosis process [4]. One of the tools that developed with modern advances today is Magnetic Resonance Imaging (MRI) [5, 6]. Magnetic resonance imaging of the knee is a non-invasive diagnostic examination to assess the anatomy and pathology of the patient's condition in the knee (joints, muscles, bones, tissues, tendons and ligaments) [7, 8].

MRI examination produces images using magnetic fields without using X-rays. MRI imaging techniques are relatively more complex and have several advantages including being able to provide images with good spatial resolution, good contrast between tissues, without ionizing radiation, and can produce images of various pieces (multi planar) namely axial, coronal, and sagittal pieces $[9,10]$. The choice of parameters will affect the quality of the image, so that the anatomy and pathology of body tissues can be carefully evaluated $[11,12]$.

Magnetic resonance imaging is good for detecting soft tissue lesions. The tissue seen on the Knee MRI examination is ACL, PCL, Meniscus, fluid, and fat [13, 14]. Knee MRI is influenced by many parameters to produce optimal images in a short time. The choice of parameters needs to consider the patient and clinical condition. Uncooperative patients tend to cause motion artifacts unless the scanning process works very fast. Artifacts on MRI are unwanted images that occur on MRI images. One of them is caused by physiological movements, due to heart movements, breathing, blood movements and movements that occur periodically such as swallowing, shaking, blinking, and others. As a result of these artifacts on MRI images will appear blurred, unclean images, visible lines and irregular images [13].

One of the parameters that makes scanning fast is the Echo Train Length (ETL) setting. Echo Train Length (ETL) shows the number of times data appears in a Time Repeatition (TR) range. Time Repetition is a parameter that controls the amount of longitudinal magnetization that is recovered before the next Radio Frequency (RF) pulse. The use of ETL variations also affects the examination time and image quality. The more ETL value increases, the faster the inspection time but the Signal to Noise Ratio (SNR) value will decrease, conversely the more ETL value decreases, the inspection time will be longer, but the SNR value increases. ETL variations really need to be done to find out at what value the ETL is optimal in order to get a good image and fast time. Range of ETL values for Turbo Spin Echo (TSE) ranging from 4 to $32[15,16]$. The most commonly used ETL in hospitals where data collection is 16 [17].

Another parameter that can accelerate and reduce motion artifacts due to movement because the patient is uncooperative is the use of the BLADE technique. The beginning of the BLADE technique used the name Periodically Rotated Overlapping Parallel Lines With Enhanched Reconstruction (PROPPELER) which was later adapted by several vendors manufacturing MRI Equipment, under the name BLADE by Siemens, PROPELLER by General Electrik, Multivane by Philips, RADAR by Hitachi and JET by Toshiba [18, 19, 20]. Blade is a technique on turbo spin echo (TSE) sequences where the tool buttons on the computer are activated by the Blade. Blade works on the system, when data acquisition is obtained then the acquisition signal is processed by fourier transformation through the reconstruction of the fast fourier transformation algorithm with the Blade radial [21]. The central signal which is the signal with the highest intensity is scattered in all directions so that the distortion effect of the movement will be covered or reduced [21]. Two parameter 
setting between ETL and Blade there are other parameters that can be combined with ETL as an option to reduce artifacts due to movement, the technique is Cartesian [22]. Cartesian technique is the process of entering data from the acquisition into $\mathrm{K}$-space following the Cartesian axis in the direction of the $\mathrm{X}$ axis along the $\mathrm{Y}$ axis, but the technique is very susceptible to movement and this is the reality in the field that the cartesian technique is used as a routine TSE sequence in knee MRI examination [23, 24]. Therefore, the writer is interested in wanting to further analyze the use of the Cartesian T2TSE and T2TSE Blade techniques combined with the selection of the right ETL parameters so as to produce a good image and fast time $[21,24,25]$.

\section{Method}

This type of research is quantitative research with an experimental study approach. Experiments were carried out to determine differences in image quality (SNR and CNR) of Knee MRI on cartesian and blade techniques with variations in ETL values and anatomical information on the Sagital T2 weighted TSE sequence, in order to obtain a short scanning time with good image quality. Data was collected at Radiology Installation at Haji Hospital Surabaya. The research sample was 10 voulunteers, each of which was carried out two treatments, namely in the T2 TSE Cartesian sequence with T2 TSE Blade. The procedure of the study was by scanning the MRI scan of the sagittal T2 TSE sequence, by adjusting the ETL parameters contained in the tool with variations of 14, 16, and 18 by combining the Cartesian T2 and T2 TSE Blade sequences. The assessment of knee MRI image results by measuring the SNR and CNR values in the area to be evaluated by adjusting the ROI in the ACL, PCL, meniscus, fluid, and fat regions. CNR measurement is done by calculating the difference in the SNR value of two adjacent tissue. CNR in this study is the difference between SNR ACL with fluid, meniscus with fluid, and PCL with fat. An assessment of anatomic information is carried out by a Radiology Specialist. Controlled variables are made constant so that they are not affected by external factors not examined in the study include TR 5450,0 ms, TE 64,0 ms, FOV $170 \mathrm{~cm}$, Receive Bandwith 150,0 Hz/px, Slice Thicknes $3 \mathrm{~mm}$, Flip Angle 150, Matriks 320x320, Interslice gap/ Distance Factor 10\%.

Data analysis was performed with Anova for SNR and CNR followed by Post Hoc test with LSD. Friedman test was conducted to find out the optimal technique to produce anatomical information clarity, then continued with the Wilcoxon test to determine the differences between the two Cartesian techniques and the Blade technique with variations in ETL with alpha 5\%.

\section{Results and Discussion}

Study was conducted on 10 male and female voulenter patients, ranging in age from 21 years to 50 years who underwent normal knee MRI examinations with TES Cartesian T2 sequences and TAG Blade sagittal pieces with 3 variations of ETL, namely 14, 16, and 18. Picture of knee MRI images on the use of a combination of cartesian techniques and techniques with ETL values as shown in Figure 1. 


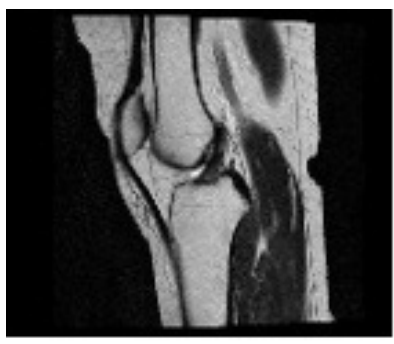

Cartesian-ETL 14

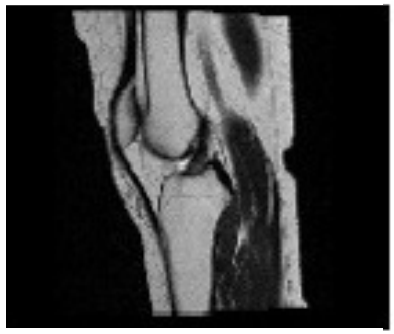

Cartesian-ETL 16

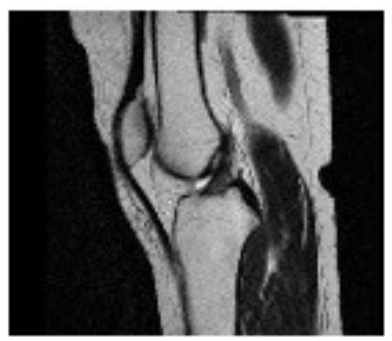

Cartesian-ETL 18

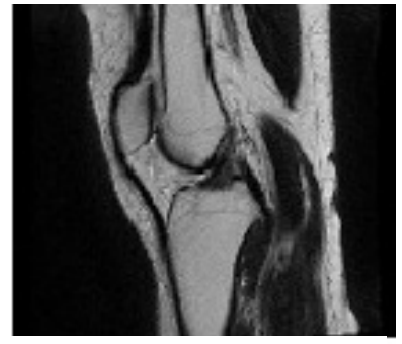

Blade-ETL 14

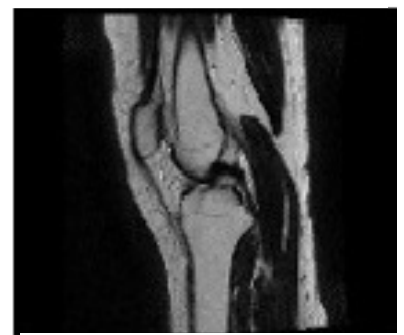

Blade-ETL 16

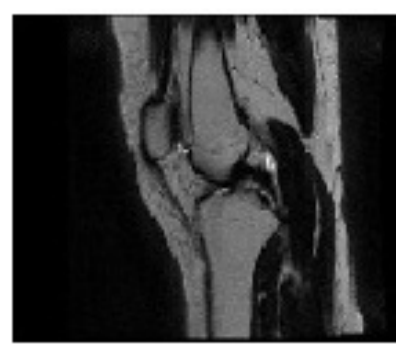

Blade-ETL 18

Figure. 1. Results of Knee MRI images with variations in Cartesian and Blade Techniques with a combination of ETL values

In the knee MRI image then the SNR and CNR values are measured with variations in ETL values on the MRI examination of the sagittal slices of the T2 TSE Cartesian sequence with the T2 Blade TSE as in table 2. The SNR measurements are carried out on the image of each ACL, PCL, Meniscus, Fluid, and Fat, which then the calculation results are averaged into Knee SNR Table 2.

Table 2. Value of SNR Knee MRI Overall Patient Image

\begin{tabular}{lc}
\hline Variable & SNR \\
\hline Cartesian-ETL 14 & $39,86 \pm 0,36$ \\
Cartesian-ETL 16 & $39,20 \pm 0,45$ \\
Cartesian-ETL 18 & $34,15 \pm 0,42$ \\
Blade-ETL 14 & $31,66 \pm 0,36$ \\
Blade-ETL 16 & $26,75 \pm 0,27$ \\
Blade-ETL 18 & $24,12 \pm 0,26$ \\
\hline
\end{tabular}


a. Differences in Image Quality (Signal to Noise Ratio) on Cartesian and Blade techniques with ETL variations.

Measurement of SNR and CNR values is done by doing ROI on the image in the MRI modality. SNR measurement is to compare the results of the average ROI measurement on the tissue (signal) with the results of the ROI measurement standard deviation in the background (noise). From the ROI placement, the SNR value can be obtained. Furthermore, the SNR value was analyzed by Anova test to find out whether there was a difference between the SNR values in variations of the combination of Cartesian and Blade Techniques for each ETL. Different Test Results as in table 3.

Table 3. Different Test of SNR between Cartesian and Blade Techniques with ETL Variations

\begin{tabular}{lll}
\hline Variable & p-value & \multicolumn{1}{c}{ Meaning } \\
\hline Cartesian-ETL 14 & & $\begin{array}{l}\text { There is a difference in the SNR } \\
\text { value for variations in the } \\
\text { Cartesian -ETL 16 }\end{array}$ \\
Cartesian-ETL 18 & 0.005 & $\begin{array}{l}\text { combination of ETL-Cartesian and } \\
\text { Blade-ETL 14 }\end{array}$ \\
Blade-ETL 16 & ETL-Blade \\
Blade-ETL 18 & & \\
\hline
\end{tabular}

Statistical test with Anova showed that there was a difference in SNR MRI Knee values between Cartesian and Blade techniques with variations in ETL values with $p$-value of $0.005(<0.05)$. To find out the difference in the quality of knee MRI images with Cartesian and Blade techniques between variations of ETL, further tests were conducted using the Post Hoc Test with LSD as shown in table 4.

Table 4. Test results for different SNR MRI Knee between Cartesian and Blade Techniques with a combination of ETL values with post hoct test

\begin{tabular}{lcl}
\hline Variable & p-value & Meaning \\
\hline Cartesian 14-Cartesian 16 & 1.000 & there is no difference of SNR \\
Cartesian 14-Cartesian 18 & 0.792 & there is no difference of SNR \\
Cartesian 14-Blade 14 & 0.455 & there is no difference of SNR \\
Cartesian 14-Blade 16 & 0.062 & there is no difference of SNR \\
Cartesian 14-Blade 18 & $\mathbf{0 . 0 1 5}$ & There is a difference SNR \\
Cartesian 16-Cartesian 18 & 0.863 & there is no difference of SNR \\
Cartesian 16-Blade 14 & 0.546 & there is no difference of SNR \\
Cartesian 16-Bade 16 & $\mathbf{0 . 0 8 5}$ & There is a difference SNR \\
Cartesian 16-Blade 18 & $\mathbf{0 . 0 2 2}$ & There is a difference SNR \\
Cartesian 18-Blade 14 & 0.993 & there is no difference of SNR \\
Cartesian 18-Blade 16 & 0.564 & there is no difference of SNR \\
Cartesian 18-Blade 18 & 0.244 & there is no difference of SNR \\
Blade 14-Bade 16 & 0.875 & there is no difference of SNR \\
Blade 14-Blade 18 & 0.564 & there is no difference of SNR \\
Blade 16-Blade 18 & 0.991 & there is no difference of SNR \\
\hline
\end{tabular}

Based on the Post Hoc Test with LSD in Cartesian ETL 14 and Blade ETL 18 there was a significant difference with a p-value of 0.015. In the comparison of Cartesian ETL 16 and Blade ETL 18 there were also significant differences with p-values of 0.022 . In the comparison of Cartesian ETL 14 and Cartesian ETL 16 there was no significant difference with p-value 1,00. Cartesian-ETL 14 and Cartesian-ETL 18, there was no significant difference with p-value of 0.792. Cartesian-ETL 14 and Blade-ETL 14 there was no significant difference with p-value of 0.455 . In the comparison of Cartesian-ETL 
14 and Blade-ETL 16 there was no significant difference with p-value of 0.062 . CartesianETL 16 and Cartesian-ETL 18 there was no difference with p-value 0.863. CartesianETL16 and Blade-ETL 14 there was no significant difference with p-value of 0.546 . Cartesian-ETL 16 and Blade-ETL 16 there was no significant difference with p-value of 0.085. Cartesian-ETL 18 and Blade ETL 14 there was no significant difference with pvalue of 0.993. In the comparison of Cartesian-ETL 18 and Blade-ETL 16 there was no significant difference with p-value of 0.564. In the comparison of Cartesian-ETL 18 and Blade-ETL 18 there was no significant difference with p-value of 0.244. Blade-ETL 14 and Blade-ETL 16 there was no significant difference with p-value 0.875. Blade-ETL 14 and Blade-ETL 18 there was no significant difference with p-value of 0.564 . Blade-ETL 16 and Blade-ETL 18 there was no significant difference with p-value of 0.991.

b. Difference in Image Quality (Contrast to Noise Ratio) Knee MRI in Cartesian and Blade techniques with ETL variations

CNR calculation is a measurement using the Region of Interest (ROI) method on two tissues signals that are close together. This CNR calculation is performed on each adjacent tissue organ images namely ACL-Fluid, Meniscus-Fluid, and PCL-Fat which then the results of the calculation are accumulated into the overall Knee CNR value of all patients which can be seen in Table 5 .

Table 5. CNR Value of Overall Patients

\begin{tabular}{lc}
\hline Variable & CNR \\
\hline Cartesian-ETL 14 & $88,69 \pm 0,63$ \\
Cartesian-ETL 16 & $88,29 \pm 0,36$ \\
Cartesian-ETL 18 & $74,80 \pm 0,27$ \\
Blade-ETL 14 & $57,70 \pm 0,35$ \\
Blade-ETL 16 & $61,77 \pm 0,54$ \\
Blade-ETL 18 & $51,66 \pm 0,36$ \\
\hline
\end{tabular}

Results measurement of CNR values in table 5 are then further analyzed using the Anova Test and Post Hoc Test to find out whether there are differences in CNR values in the variation of Cartesian and Blade combinations for each ETL.

Table 6. Different Test of CNR between Cartesian Technique and Blade Technique with ETL Variation

\begin{tabular}{lll}
\hline \multicolumn{1}{c}{ Variable } & p-value & \multicolumn{1}{c}{ Meaning } \\
\hline Cartesian-ETL 14 & & There Are Different CNR \\
Cartesian-ETL 16 & & $\begin{array}{l}\text { Values in ETL-Cartesian and } \\
\text { Cartesian-ETL 18 }\end{array}$ \\
Blade-ETL 14 & 0.005 & $\begin{array}{l}\text { ETL-Blade Combination } \\
\text { Blade-ETL 16 }\end{array}$ \\
Blade-ETL 18 & & Variations \\
\hline
\end{tabular}

Test results show that there is a difference in MRI Knee image quality (CNR) between Cartesian and Blade techniques with ETL variations with a p-value of $0.005(<0.05)$. To find out the difference in image quality (CNR) of MRI knee between the Cartesian technique and the Blade technique in every variation of ETL, a follow-up test was done using the Post Hoc Test with LSD as shown in table 7. 
Table 7. Different Test results for CNR between Cartesian and Blade Techniques with ETL Variations with the Post Hoc LSD Test

\begin{tabular}{lcl}
\hline \multicolumn{1}{c}{ Variable } & p-value & \multicolumn{1}{c}{ Meaning } \\
\hline Cartesian 14-Cartesian 16 & 1.000 & there is no difference of CNR \\
Cartesian 14-Cartesian 18 & 0.734 & there is no difference of CNR \\
Cartesian 14-Blade 14 & 0.692 & there is no difference of CNR \\
Cartesian 14-Blade 16 & 0.106 & there is no difference of CNR \\
Cartesian 14-Blade 18 & $\mathbf{0 . 0 1 0}$ & There is a difference of CNR \\
Cartesian 16-Cartesian 18 & 0.805 & there is no difference of CNR \\
Cartesian 16-Blade 14 & 0.767 & there is no difference of CNR \\
Cartesian 16-Bade 16 & 0.138 & there is no difference of CNR \\
Cartesian 16-Blade 18 & $\mathbf{0 . 0 1 4}$ & There is a difference of CNR \\
Cartesian 18-Blade 14 & 1.000 & there is no difference of CNR \\
Cartesian 18-Blade 16 & 0.779 & there is no difference of CNR \\
Cartesian 18-Blade 18 & 0.218 & there is no difference of CNR \\
Blade 14-Bade 16 & 0.816 & there is no difference of CNR \\
Blade 14-Blade 18 & 0.247 & there is no difference of CNR \\
Blade 16-Blade 18 & 0.911 & there is no difference of CNR \\
\hline
\end{tabular}

After the Post Hoc Test done, the comparison of Cartesian-ETL 14 and Blade-ETL 18 there is a significant difference with $p$-value $=0.010$. Cartesian-ETL 16 and BladeETL 18 there were also significant differences with p-values of 0.014. Cartesian-ETL 14 and Cartesian- ETL 16 there was no significant difference with p-value 1,00. CartesianETL 14 and Cartesian-ETL 18 there was no significant difference with p-value of 0.734 . Cartesian-ETL 14 and Blade-ETL 14 there was no significant difference with p-value of 0.692. Cartesian-ETL 14 and Blade-ETL 16 there was no significant difference with pvalue of 0.106. Cartesian-ETL 16 and Cartesian-ETL 18 there was no difference with pvalue of 0.805. Cartesian-ETL 16 and Blade-ETL 14 there was no significant difference with p-value of 0.767. Cartesian-ETL 16 and Blade-ETL 16 there was no significant difference with p-value of 0.138. Cartesian-ETL 18 and Blade-ETL 14 there was no significant difference with p-value 1.00. Cartesian-ETL 18 and Blade-ETL 16 there was no significant difference with p-value of 0.779. Cartesian-ETL 18 and Blade-ETL 18 there was no significant difference with p-value of 0.218 . Blade-ETL 14 and Blade-ETL 16 there was no significant difference with p-value 0.816. Blade-ETL 14 and Blade-ETL 18 there was no significant difference with p-value of 0.247 . Blade-ETL 16 and BladeETL 18 there was no significant difference with p-value of 0.91 .

c. Differences Image Anatomy Information in Knee MRI on Cartesian and Blade techniques with ETL variations

Clarity of anatomic information was obtained from the assessment of a radiology sepsialist doctor with control by an equivalent respondent. The results of the alignment assessment perception between respondents as shown in table 8 .

Table 8. Appropriateness Test of evaluation among respondents

\begin{tabular}{ccc}
\hline Variable & Koef-Kappa & p-value \\
\hline $\begin{array}{c}\text { Responden 1 - } \\
\text { Responden 2 }\end{array}$ & 0,8712 & $<0,001$ \\
\hline
\end{tabular}

Results of the appropriateness test between respondents showed equality of perception with kappa coefficient $=0.8712$ with $p$-value $<0.001$. To find out the differences Anatomy Information in Knee MRI on Cartesian and Blade techniques 
with ETL variations, the Friedman Test and Wilcoxon Test, as shown in tables 9 and 10. Different tests with Friedman-test are used to determine differences in anatomic information between Cartesian and Blade techniques with variations ETL value.

Table 9. Results of Anatomical Information Difference Test between Cartesian and Blade Technique with Friedman Test

\begin{tabular}{lccl}
\hline \multicolumn{1}{c}{ Variabel } & p-value & Mean Rank & \multicolumn{1}{c}{ Meaning } \\
\hline Cartesian-ETL 14 & & 4.58 & There are differences in \\
Cartesian-ETL 16 & & 4.23 & anatomic information on \\
Cartesian-ETL 18 & $<0.001$ & 2.37 & variations in ETL-Cartesian \\
Blade-ETL 14 & & 4.75 & and ETL-Blade \\
Blade-ETL 16 & & 2.82 & Combinations \\
Blade-ETL 18 & & 2.25 & \\
\hline
\end{tabular}

Friedman test results in the table show a significant difference in anatomic information between cartesian and Blade techniques using variations in ETL values with p-values $<0.001(<0.05)$. Based on anatomic information that produces the best image quality is the Blade technique with ETL 14, with the highest mean rank value of 4.75 which shows that the use of these techniques is the most optimal for showing anatomic information.

Wilcoxon Test is then performed to determine the differences in each variation. Wilcoxon test is used to determine the differences in each Cartesian and Blade technique with ETL variations as shown in table 10.

Table 10. Results of Difference Test of Anatomical between Cartesian and Blade Techniques with ETL Variations using the Wilcoxon Test

\begin{tabular}{|c|c|c|}
\hline Variabel & p-value & Keterangan \\
\hline Cartesian 14-Cartesian 16 & 0.072 & there is no difference in anatomic information \\
\hline Cartesian 14-Cartesian 18 & 0.043 & there is a difference in anatomic information \\
\hline Cartesian 14-Blade 14 & 0.414 & there is no difference in anatomic information \\
\hline Cartesian 14-Blade 16 & $<0.001$ & there is a difference in anatomic information \\
\hline Cartesian 14-Blade 18 & $<0.001$ & there is a difference in anatomic information \\
\hline Cartesian 16-Cartesian 18 & 0.180 & there is no difference in anatomic information \\
\hline Cartesian 16-Blade 14 & 0.014 & there is a difference in anatomic information \\
\hline Cartesian 16-Blade 16 & 0.004 & there is a difference in anatomic information \\
\hline Cartesian 16-Blad & $<0.001$ & there is a difference in anatomic information \\
\hline Cartesian 18-Blade 14 & $<0.001$ & there is a difference in anatomic information \\
\hline Cartesian 18-Blade 16 & 0.119 & there is no difference in anatomic information \\
\hline Cartesian 18-Blade 18 & 0.763 & there is no difference in anatomic information \\
\hline Blade 14-Blade 16 & 0.067 & there is no difference in anatomic information \\
\hline Blade 14-Blade 18 & 0.021 & there is a difference in anatomic information \\
\hline Blade 16-Blade 18 & 0.081 & there is no difference in anatomic information \\
\hline
\end{tabular}

Based on these data, there is no significant difference in the comparison of Cartesian-ETL 14 and Blade-ETL 14 with a p-value of 0.414. Cartesian-ETL 16 and Blade-ETL 14 there is a significant difference with p-value of 0.014. Cartesian-ETL 18 and Blade-ETL 14 there was a significant difference with $p$-value $<0.001$. Cartesian-ETL 14 and Blade-ETL 16 there were significant differences with $\mathrm{p}$-values $<0.001$. Cartesian-ETL 16 and Blade-ETL 16 there is a significant difference with p-value 0.004. Cartesian-ETL14 and Blade-ETL 18 there were significant differences with $p$-values $<0.001$. Cartesian-ETL 16 and Blade-ETL 18 there was 
a significant difference with p-value $<0.001$. Cartesian-ETL 14 and Blade-ETL 14 there was no significant difference with p-value 0.414. Cartesian-ETL 18 and Blade-ETL 16 there was no significant difference with p-value of 0.119. Cartesian-ETL 18 and Blade-ETL 18 there was no significant difference with p-value of 0.763 .

d. The best technique for producing image quality and anatomic information on Knee MRI in Cartesian and Blade techniques with ETL variations.

1. Base on quality image (SNR and CNR)

Result of Statistical Tests show that the highest SNR value in both techniques and produce the best Knee MRI image quality is Cartesian technique, ETL 14 with a value of 39.86

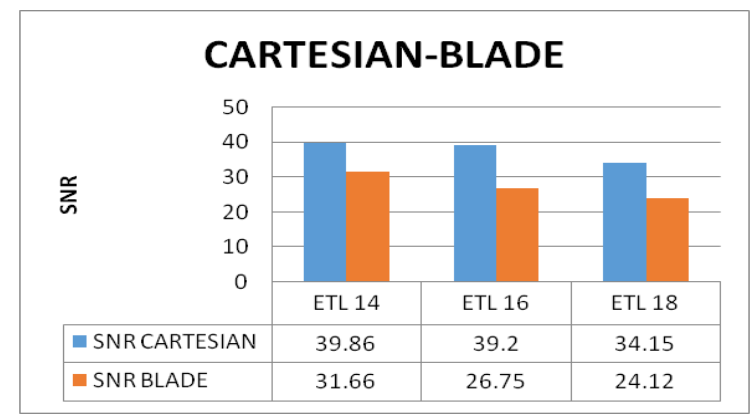

Fig. 1. SNR value of Cartesian and Blade Techniques with ETL Variation

For the CNR assessment, the statistical test results showed that the highest CNR value in the two techniques that produced the best Knee MRI image quality was the Cartesian technique, ETL 14 with a value of 88.69 .

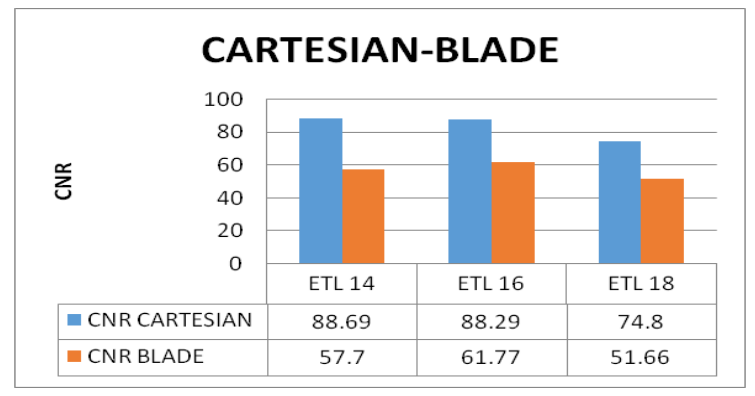

Fig. 2. CNR Values of Both Cartesian and Blade Techniques with ETL Variation

2. Base on Anatomic Information

Result of the statistical tests in table 8 , it shows the highest mean rank value in the use of the Blade technique with ETL 14, this shows that the use of the Blade technique with ETL 14 with a mean rank value of 4.75 is the most optimal and provides more superior information than with other parameters.

\section{DISCUSSION}

1. The difference in the quality of the Knee MRI image to Cartesian and Blade techniques with variations in ETL values.

a. The difference in the value of image quality (SNR and CNR) on Cartesian and Blade techniques with variations in ETL values 
The difference to the SNR value can be seen in graph 1. From the graph it is known that the relationship between the use of Cartesian and BLADE techniques with the provision of ETL variations. In general, Cartesian is superior to BLADE in terms of displaying SNR. ETL 14 has the best image quality compared to 16 and 18 . The long echo train on T2 FSE MRI weighting can cause blurring, affecting the MRI image quality, which allows reducing the SNR value and certainly will affect the CNR, this is one of the weaknesses of long ETL $[22,24]$. Excessive ETL applications cause the signal amplitude to decrease because ETL applications are large enough with Time Echo (TE) which increasingly leads to phase coding to fill K-Space faster. The result, can reduce the signal amplification occurs but the scanning time will be faster [21, 25]. The greater the ETL, the shorter the scan time. Because the K-space coding process will be faster, causing scanning time to be faster too, but the consideration in getting diagnostic information is not only due to the speed of the scan time, but how it can produce high-quality image quality, and in this case ETL 14 which produces high SNR value [25].

The difference to the CNR value can be seen in graph 2, from the graph shows the relationship between the use of the Cartesian technique and the BLADE technique with the same ETL variations. Graph 2 shows the CNR values in both Cartesian and Blade techniques by giving the same ETL variations, that the Cartesian technique with ETL 14 gives optimal image quality results, compared to ETL 16 and 18. The difference in Cartesian and Blade techniques is due to differences in data acquisition to $\mathrm{K}$ - space which is a space where signals in the form of frequencies originating from patients are stored. In the Cartesian technique the process of entering data from the acquisition into the $\mathrm{K}$-space follows the Cartesian axis in the direction of the $\mathrm{X}$ axis along the $\mathrm{Y}$ axis, so that the signal results are spread evenly throughout the K-space area. Whereas in the Blade technique the process of entering data from acquisition into K-space is processed using fourier transforms through the reconstruction of the algorithm fast fourier transformation with radial Blade meaning the central area signal (center) with the highest intensity is scattered in all directions so that the distortion effect of the movement is covered or reduced [20,21].

The advantage of the Cartesian technique compared to the Blade technique is in terms of time, because when encoding data into K-space the Cartesian technique is faster than the Blade technique. Variation in ETL values can affect the image quality of the Cartesian and Blade techniques but there is no significant difference because in each echo range it has a different phase enchode for each TR but will fill the lines on the K-spacenya similarly in image formation $[15,20]$

Long ETL in weighting T2 MRI Knee TSE can cause blurring that affects SNR and CNR signals which is a weakness of TSE. So that the Cartesian T2 TSE and T2 TSE Blade techniques have significant differences [16, 24].

b. Differences in Cartesian and Blade techniques with ETL variations on anatomic information

The difference in image quality with anatomic information in Cartesian and Blade techniques with ETL variations is the Blade ETL 14 technique because it is indicated by a large mean rank value. due to the influence of the use of BLADE trajectory which aims to reduce artifacts running well in suppressing artifacts. According to Elliott artifacts that appear can interfere with anatomical imagery and can cause inaccuracies in making a diagnosis [25]. The emergence of artifacts in the Cartesian sequence is what reduces the value of anatomic information, resulting in anatomical information differences between Cartesian and Blade. Differences in anatomical information between Cartesian and BLADE on knee MRI examination can also be caused by the influence of different sequential parameters used respectively. The use of Cartesian 
parameters is a parameter used in hospitals while the BLADE parameter is the adoption of a Cartesian only in the trajectory system changed from Cartesian to BLADE with the consequence of changes in other parameters namely time echo (TE), avareges (NEX) and Elliptical Filter from on to off [20, 25].

2. The best technique to produce Knee MRI image quality in Cartesian and Blade techniques with ETL variations

a. Consideration of aspects of image quality (SNR and CNR)

Knee MRI image quality with ETL variation can be seen from the SNR value that the best technique to produce optimal images is Cartesian technique with ETL 14, because the smaller the ETL value used in MRI images, the higher the SNR value. Otherwise if the ETL value increases, the smaller the SNR value obtained. To evaluate image quality is very subjective, depending on the respondent's vision. Some people may not be able to see high background noise if the resolution in the area is high. On the other hand only wants a high SNR even though the resolution is low. Patients also affect the onset of noise. Every time a proton changes (down) from high energy to low energy, it emits photons (radio waves) with a random phase and will contribute to background noise. This can also be possible if there is a movement in the patient, where the activity changes in energy will impact on the contribution of noise to the MRI image [17, 25].

Knee MRI image quality can be seen from both the Cartesian and Blade techniques with variations ETL on the CNR value that the best technique is the Cartesian technique with ETL 14, because the contrast will be clearly seen. Then if seen from the variable ETL the higher the ETL value used, the image contrast will be worse. A good CNR can show the difference between normal and abnormal areas both anotomically and pathologically. This difference is influenced by MRI parameters. Difference in signal differences between the two tissues against noise will affect the results of the contrast value by showing a clear contrast difference on MRI imaging. The greater the CNR value, image contrast quality will be better $[21,25]$

b. Consideration of aspects of Anatomic Information

Knee MRI image quality on both Cartesian and Blade techniques with ETL variations on anatomic information that the best technique is the Blade technique with ETL 14, this is because the Blade technique is able to reduce motion artifacts so that the distortion effects of movement can be covered or reduced.

In making decisions based on three considerations, SNR, CNR, and anatomical information, researchers choose the Blade technique with ETL 14 as the best technique for obtaining MRI Knee image quality and establish diagnosis.

From the Wilcoxon test results on anatomic information, Cartesian comparison with ETL 14 and Blade with ETL 14 produces a p-value of $0.414(>0.05)$, so there is no significant difference between cartesian with ETL14 and Blade with ETL 14. From the Wilcoxon test results for anatomic information, the comparison of Cartesian with ETL 14 and Blade with ETL 14 produces a p-value of 0.414 ( $>0.05)$, so there is no significant difference between cartesian with ETL14 and Blade with ETL 14.

\section{Conclusion and Suggestions}

Based on research that has been done, it can be concluded that:

a. There is a difference between SNR and CNR Knee MRI using Cartesian and BLADE technique by giving variations of ETL values with p-value 0.005 .

b. There are differences in anatomical information on Knee MRI images using Cartesian and BLADE techniques with variations in ETL values with p-values $<0.001$ 
c. The best technique to produce SNR and CNR MRI Knee with ETL variations is Cartesian technique with ETL value of 14 .

d. The best technique to produce good anatomical information is the Blade technique with ETL value of 14 .

e. There is no difference in image quality between the Cartesian ETL 14 and the Blade ETL 14 technique with a p-value of 0.414 . So that both techniques can be used as an alternative to the Knee MRI examination.

f. In making the best technique and parameter conclusions on Knee MRI T2 TSE examination, although the highest quality factor is Cartesian technique with ETL 14, but the preferred technique is Blade, ETL 14 with consideration of aspects of diagnosis and can display superior anatomical information.

\section{References}

[1] Salem, K. A. 1999. MRI Hot Topics Motion Correction for MR Imaging s medical.

[2] Prestasi, J. O. (2016). Jurnal Olahraga Prestasi, Volume 12, Nomor 2, Juli 2016 | 41, $12,41-60$.

[3] Notosiswoyo, Mulyono, 2004, Media LitbangKesehatanPemanfaatan Magnetic Resonance Imaging (MRI) SebagaiSarana DiagnosaPasien, Volume XIV, Nomer 3

[4] Hashemi, H. Ray and Bradley, G. William, 1997, MRI: The Basic, Williams \& Wilkins, USA

[5] Woodward, Peggy ang William, W. Arrison, 1997, MRI Optimization, a hand on approach, McGraw-Hill, Co. USA

[6] W. McRobbie, E. A. Moore, M. J. Graves and M. R. Graves, MRI From Picture to Proton, Second Edition. Cambridge, New York

[7] Rodriguez, Jr., 2007, MRI Appearance of Posterior Cruciate Ligament Tears, Am J of Roentgenology 2008.191:W155-W159

[8] Alibek S, Adamietz B, Cavallaro A, et al. Contrast enhanced T1-weighted fluidattenuated inversion-recovery BLADE magnetic reso-nance imaging of the brain: an alternative to spin-echo technique for detection of brain lesions in the unsedatedpediatric patient? AcadRadiol 2008;15:986-95

[9] Weishaupt.Dominik, D.Kochli.Victor, Marincek.Borut. 2006. How Does MRI Work? An Introduction the Physic and Function of Magneting Resonance Imaging.Heildelberg: Business Media.

[10] Bencardino, 2009, MR Imaging of Complications of Anterior Cruciate Ligament Graft Reconstruction, CRSNA, $2009 \cdot$ radiographics.rsna.org.

[11] Bushberg J.T., Seibert J.A., Edwin M. Leidholdt, J.R., Boone J.M., 2002, The Essential Physic of Medical Imaging, Second Edition, Lippincott Williams \& Wilkins, Philadelphia USA.

[12] Bjørnerud, Atle. 2008. The Physics of Magnetic Resonance Imaging. Department of Physics University of Oslo.

[13] Currie, Stuart, et al. 2015.Understanding MRI: basic MR physics for physicians.Postgrad Med J

[14] Rochmayanti, D., Widodo, T. S., \& Soesanti, I. (2013). Analisis Perubahan Parameter Number of Signals Averaged ( NSA ) Terhadap Peningkatan SNR dan Waktu Pencitraan pada MRI, 2(4).

[15] Westbrook, Catherine and Kaunt, Caroline. 2011. MRI in Practice Fourth Edition.

[16] Mag ResonPencitraan 2003; 21: 745-753. Sze G, Kawamura Y, Negishi C, et al. Cepat spin-echo MR Pencitraandaritulangbelakangleher: pengaruh echo train length danjarakgemapadakontrasgambardankualitas 
[17] Hornak, J.P., 1996-2011, The Basic of MRI, imaging Hardware, http://www.cis.rit.edu/htbooks/mri/chap-9/chap-9htm

[18] H.Schild, Hans. 1990. MRI Made Easy.Berlin :CopyrightbyScheringAG.

[19] Ii, B. A. B., \& Teori, L. (1977). Universitas Sumatera Utara, 4-24. J.Blink,Evert. 2004. Basic MRI : Physics. Netherlands

[20] Lavdas E, Mavroidis P, Hatzigeorgiou V, Roka V, ArikidisN, Oikonomou G, et al. Elimination of motion and pulsation artifacts using BLADE sequences in knee MR imaging. MagnReson Imaging 2012 In press.

[21] Pipe, J. G. (1999). Motion Correction With PROPELLER MRI : Application to Head Motion and Free-Breathing Cardiac Imaging, 969, 963-969.

[22] Siemens. 2010. Physical Principles, CS Training Center MR Basics All rightsreserved. Copyright $(\odot)$ Siemens AG

[23] Pipe, J. G. (1999). Motion Correction With PROPELLER MRI : Application to Head Motion and Free-Breathing Cardiac Imaging, 969, 963-969.

[24] Symons, F. S., Nelson, F. A., \& Roberts, T. P. (2006). MR Pulse Sequences: What Every Radiologist Wants to Know but Is Afraid to Ask 1, 2, 513-538.

[25] Josepa ND, Simanjuntak, dkk, Studi Analisis Echo Train Length Dalam K-Space Serta Pengaruhnya Terhadap Kualitas Citra Pembobotan T2 FSE Pada MRI 1,5 T, Berkala Fisika Vol 17 No. 1 Januari 2014, hal 7-12. ISSN 1410-9662 